\title{
Intensive post-operative follow-up of breast cancer patients with tumour markers: CEA, TPA or CAI5.3 vs MCA and MCA-CAI5.3 vs CEA-TPA-CAI5.3 panel in the early detection of distant metastases
}

\author{
Andrea Nicolini*1, Gianna Tartarelli1 ${ }^{1}$, Angelo Carpi ${ }^{2}$, Maria Rita Metelli ${ }^{3}$, \\ Paola Ferrari ${ }^{1}$, Loretta Anselmi ${ }^{1}$, Massimo Conte ${ }^{4}$, Piero Berti ${ }^{4}$ and \\ Paolo Miccoli ${ }^{4}$
}

Address: ${ }^{1}$ Department of Internal Medicine, University of Pisa, Pisa, Italy, ${ }^{2}$ Department of Reproduction and Ageing, University of Pisa, Pisa, Italy, ${ }^{3}$ Department of Experimental Pathology, University of Pisa, Pisa, Italy and ${ }^{4}$ Department of Surgery, University of Pisa, Pisa, Italy

Email: Andrea Nicolini* - a.nicolini@int.med.unipi.it; Gianna Tartarelli - a.nicolini@int.med.unipi.it;

Angelo Carpi - a.nicolini@int.med.unipi.it; Maria Rita Metelli - a.nicolini@int.med.unipi.it; Paola Ferrari - a.nicolini@int.med.unipi.it; Loretta Anselmi - a.nicolini@int.med.unipi.it; Massimo Conte - a.nicolini@int.med.unipi.it; Piero Berti - a.nicolini@int.med.unipi.it; Paolo Miccoli - a.nicolini@int.med.unipi.it

* Corresponding author

Published: 20 November 2006

BMC Cancer 2006, 6:269 doi:10.1 186/147|-2407-6-269
Received: 08 November 2005

Accepted: 20 November 2006

This article is available from: http://www.biomedcentral.com//47/-2407/6/269

(C) 2006 Nicolini et al; licensee BioMed Central Ltd.

This is an Open Access article distributed under the terms of the Creative Commons Attribution License (http://creativecommons.org/licenses/by/2.0), which permits unrestricted use, distribution, and reproduction in any medium, provided the original work is properly cited.

\footnotetext{
Abstract

Background: In breast cancer current guidelines do not recommend the routine use of serum tumour markers. Differently, we observed that CEA-TPA-CA I5.3 (carcinoembryonic (CEA) tissue polypeptide (TPA) and cancer associated II5D8/DF3 (CAI5.3) antigens) panel permits early detection and treatment for most relapsing patients. As high sensitivity and specificity and different cut-off values have been reported for mucin-like carcinoma associated antigen (MCA), we compared MCA with the above mentioned tumour markers and MCA-CAI5.3 with the CEA-TPA-CAI5.3 panel.

Methods: In 289 breast cancer patients submitted to an intensive post-operative follow-up with tumour markers, we compared MCA (cut-off values, $\geq I I$ and $\geq I 5 \mathrm{U} / \mathrm{mL}$ ) with CEA or CAI5.3 or TPA for detection of relapse. In addition, we compared the MCA-CAI5.3 and CEA-TPA-CAI5.3 tumour marker panels.

Results: Distant metastases occurred 19 times in 18 (6.7\%) of the 268 patients who were disease-free at the beginning of the study. MCA sensitivity with both cut-off values was higher than that of CEA or TPA or CAI $5.3(68 \%$ vs $10 \%, 26 \%, 32 \%$ and $53 \%$ vs $16 \%, 42 \%, 32 \%$ respectively). With cut-off $\geq I I \mathrm{U} / \mathrm{mL}$, MCA showed the lowest specificity (42\%); with cut-off $\geq 15 \mathrm{U} / \mathrm{mL}$, MCA specificity was similar to TPA (73\% vs $72 \%)$ and lower than that of CEA and CAI5.3 $(96 \%$ and $97 \%$ respectively). With $\geq 15 \mathrm{U} / \mathrm{mL}$ MCA cut-off, MCA sensitivity increased from $53 \%$ to $58 \%$ after its association with CAI5.3. Sensitivity of CEA-TPA-CAI5.3 panel was 74\% (14 of 19 recurrences). Eight of the 14 recurrences early detected with CEA-TPA-CAI5.3 presented as a single lesion (oligometastatic disease) (5) or were confined to bony skeleton (3) (26\% and $16 \%$ respectively of the 19 relapses). With $\geq 1 \mathrm{I} \mathrm{U/mL} \mathrm{MCA} \mathrm{cut-off,} \mathrm{MCA-CAI5.3} \mathrm{association} \mathrm{showed} \mathrm{higher} \mathrm{sensitivity} \mathrm{but}$ lower specificity, accuracy and positive predictive value than the CEA-TPA-CAI5.3 panel.

Conclusion: At both the evaluated cut-off values serum MCA sensitivity is higher than that of CEA, TPA or CAI5.3 but its specificity is similar to or lower than that of TPA. Overall, CEA-TPA-CAI5.3 panel is more accurate than MCA-CAI5.3 association and can "early" detect a few relapsed patients with limited metastatic disease and more favourable prognosis. These findings further support the need for prospective randomised clinical trial to assess whether an intensive postoperative follow-up with an appropriate use of serum tumour markers can significantly improve clinical outcome of early detected relapsing patients.
} 


\section{Background}

In breast cancer patients, current guidelines post-operatively recommend mammography at regular intervals and not routine use of any instrumental or laboratory test for early detection of relapse and monitoring of metastatic disease. In fact, in randomised trials and meta-analysis intensive post-operative follow-up has been shown to be useful only in early diagnosis but not in improving clinical outcome and/or quality of life [1-4]. Nevertheless, in these trials clinical-instrumental was compared with clinical only follow-up and neither any serum tumour marker panel nor appropriate criteria for its use were adopted. Different studies appropriately using serum tumour markers within an intensive post-operative follow-up showed that in many relapsing patients clinical-instrumental diagnosis was anticipated and that this anticipation permitted an earlier treatment which significantly prolonged disease-free survival (DFS) and/or overall survival (OS) [58]. Moreover we showed that this type of follow-up strongly reduced need for conventional radiological examinations [9-11]. Therefore, unlike current guidelines, it is routine practice in our center to carry out an intensive post-operative follow-up of breast cancer patients using both serum tumour markers and imaging techniques.

Carcinoembryonic (CEA) and breast cancer associated 115D8/DF3 (CA15.3) antigens are the serum tumour markers commonly used for post-operative monitoring of breast cancer [1] although many other tumour markers have been investigated [12-17]. We reported similar sensitivity for TPA and CA15.3, which, however, is higher than that of CEA. TPA showed much lower specificity than CA15.3 and CEA. The association of these three markers increased sensitivity with a slight decrease of specificity [4$6,18]$.

CA15.3 is one of the mucin-like biomarkers which also recently have been reported among the most useful markers to detect and monitor metastatic breast cancer $[8,19]$. The mucin-like carcinoma associated antigen (MCA) is another widely used test to assay MUC-1. When it has been used alone, high sensitivity and specificity have been reported $[17,20,21]$. This suggests the association with CA15.3. However, different cut-off values have been reported for MCA $[17,20]$. Besides conflicting data have been found both as to MCA sensitivity and specificity compared to CA15.3 [22-25] and the MCA-CA15.3 usefulness $[14,15,22,26,27]$. Finally, as far as we know, no previous study compared MCA-CA15.3 to CEA-TPACA15.3 association. Therefore, in this study we compared sensitivity and specificity of MCA (with two commonly used cut-off values: $\geq 11$ and $\geq 15 \mathrm{UI} / \mathrm{mL}$ ) with that of CEA, CA15.3, TPA for early detection of relapse. Moreover, also we compared the diagnostic accuracy and predic- tive value of the MCA-CA15.3 association to that of CEATPA-CA15.3 panel.

\section{Methods \\ Patients' follow-up}

From March 2000 to September 2003, 289 breast cancer patients aged 27 to 80 years (median 51) were submitted to an intensive post-operative follow-up with serial serum determination of CEA, CA15.3, TPA and MCA. At entry 268 patients (93\%) were disease-free (M0) and 21 (7\%) showed distant metastases (M1). At the post-operative histology $83(31 \%)$ of the 268 disease-free patients were $\mathrm{N}+$ while the 185 remaining were $\mathrm{N}$-. Premenopausals were 162 (56\%) of all the 289 studied patients. Soon after primary surgery, all estrogen (ER) and/or progesterone receptor(PgR) positive patients received hormone therapy. Moreover, all N+ and $129 \mathrm{~N}$ - disease-free patients, consistent with the current international guidelines, received adjuvant chemotherapy. As to the interval time of post-operative monitoring, patients were divided into 2 groups: at low and at intermediate-high risk of recurrence according to whether they were N-PgR+ or N+ and/or PgR$(\mathrm{N}+\mathrm{PgR}+, \mathrm{N}+\mathrm{PgR}-, \mathrm{N}-\mathrm{PgR}-)$ respectively. Axillary lymphnodes $(\mathrm{N}+/ \mathrm{N}-)[28]$ and progesterone ( $\mathrm{PgR}+/ \mathrm{PgR}-)$ status $[29,30]$ were used to divide patients into two different risk groups as they are commonly reported among the principal prognostic factors for relapse. The 126 low risk patients underwent control visits every 6 months and the remaining 142 with intermediate-high risk of recurrence every 4 months. Post-operative follow-up was $16 \pm 8$ months ( $\mathrm{m} \pm \mathrm{sd}$; range 6-37 months). At each control visit, history, routine lab and serum CEA, CA15.3, TPA and MCA measurement were carried out.

Baseline Sx-ray was performed to identify benign lesions due to inflammatory and/or degenerative disease. As to other conventional instrumental examinations, bone scintigraphy (BS) and liver echography (LE) have been reported to be more accurate than chest $\mathrm{x}$-ray (CXR) to "early" detect recurrences $[7,9,10,16,31,32]$. Therefore, BS and LE were serially performed every 24-30 months and CXR at more prolonged intervals (mean value 42 months).

The reason for serial BS, LE and CXR examinations, even if more prolonged, was to detect asymptomatic relapses falsely negative with serum tumour markers, which as we have previously reported $[4,7,9-11]$ are about $15-25 \%$ using CEA-TPA-CA15.3 tumour marker panel.

Patients suspected of relapse with CEA-TPA-CA15.3 tumour marker panel immediately underwent the standard radiological examinations (BS, LE and CXR). If these examinations were pathological or equivocal, patients immediately were selected to be further investigated as 
follows. All hot spots on the bone scintigraphy with an equivocal interpretation were examined by computed tomography and/or magnetic resonance imaging (MRI). The lesions that were considered equivocal by conventional chest $\mathrm{x}$-ray were clarified by computed tomography or bronchoscopy and cytologic study. The lesions felt to be equivocal at liver echography were clarified by computed tomography or fine needle aspiration cytology guided by liver echography when possible. The patients with equivocal standard radiological examinations and concomitantly not suspected of relapse with the CEA-TPACA15.3 tumour marker panel, were regularly followed-up as above described.

All patients gave their consent to be post-operatively monitored with all instrumental and laboratory examinations described in the paper.

\section{Tumour markers}

Serum CEA, TPA, CA15.3 and MCA concentrations were measured in fasting patients by commercial immunoenzimatic assays: Abbott, Rome (Italy) for CEA and CA15.3; DRG, Marburg (Germany) for TPA; Roche Diagnostics, Manheim (Germany) for MCA. The within and between assay coefficients of variation for CEA, CA15.3, TPA and MCA were all less than $5 \%$ and $6 \%$ respectively. Serum levels $>7 \mathrm{ng} / \mathrm{mL}$, $>95 \mathrm{U} / \mathrm{L}$ and $>32 \mathrm{U} / \mathrm{mL}$ were considered to be elevated for CEA, TPA and CA15.3 respectively; for MCA, $\geq 11$ and $\geq 15 \mathrm{U} / \mathrm{mL}$ cut-off values were considered. We identified the causes of false positive tumour marker increase [17]. As previously described $[4,9,10]$, a dynamic evaluation of tumour markers was made and in cases of a high tumour marker value a further blood sample was drawn two weeks to a month after the previous elevated value. If the re-measured tumour marker value had decreased to a normal value, the initial elevated value was considered to be an isolated elevated value (IEV). The elevated tumour marker was considered to be progressive (PI) when it was $30 \%$, or more, higher in the sample which followed the initial elevated value. Otherwise, two equally high values were regarded to be a constant elevation (CE). Only CE and/or PI were considered a significant tumour marker increase.

As previously reported [9-11] in our clinical practice only patients with CE or PI in one or more tumour markers, clearly unexplained by any other condition, are considered suspected of tumour relapse.

Tumour marker lead time was the time from the suspicion of relapse with serum tumour marker to confirmation of relapse by radiological examinations. When a clinically disease-free patient was suspected of relapse by re-testing of tumour markers at the regular control visit, 15 to 30 days were necessary to carry out the common (bone scin- tigraphy, liver echography, chest x-ray) and in case of their equivocal result, more accurate radiological examinations (CT, MRI) to confirm or rule out the suspicion. Radiological investigations performed during this 15 to 30 days interval time and confirming the initial suspicion by tumour markers were considered as they had been performed at the same time of tumour marker re-testing; therefore in this case tumour marker lead time was computed as zero. When a patient became suspected of metastases by symptoms before the routine testing of serum tumour markers (i.e. in the interval between two regular control visits), at this time immediately the entire planned procedure was carried out to confirm or rule out the suspicion. Again, as above mentioned, the time necessary for the entire procedure took about 15 to 30 days and this interval time was not considered for the calculation of the tumour marker lead time. In fact, when suspicion of metastases was confirmed by radiological examinations and not by tumour marker panel, tumour marker panel was considered falsely negative. When suspicion of metastases was confirmed by radiological examinations and by tumour marker panel as well, the tumour marker panel lead time was considered zero if symptoms suspicious of metastases had appeared at the same time the entire procedure for confirmation was started; if symptoms suspicious of metastases had previously appeared, clinical symptoms only were considered the first signal of relapse and tumour marker panel was considered falsely negative.

\section{Statistical analysis}

Sensitivity was defined as $\mathrm{TP} /(\mathrm{TP}+\mathrm{FN}) \%$ specificity as $\mathrm{TN} /$ $(\mathrm{TN}+\mathrm{FP}) \%$, accuracy as $\mathrm{TN}+\mathrm{TP} /(\mathrm{TN}+\mathrm{FN}+\mathrm{TP}+\mathrm{FP})$, positive predictive value as $\mathrm{TP} /(\mathrm{TP}+\mathrm{FP})$, negative predictive value as $\mathrm{TN} /(\mathrm{TN}+\mathrm{FN})$, where $\mathrm{FP}=$ false positive, $\mathrm{FN}=$ false negative, $\mathrm{TP}=$ true positive, $\mathrm{TN}=$ true negative. Two analyses were performed. In a first analysis only dynamic evaluation of tumour markers was taken into account without considering clinical and laboratory data. In this analysis tumour marker assay with CE or PI in one or more markers was defined as a probably positive test. In a further analysis dynamic evaluation of tumour markers was considered with an accurate clinical history and laboratory examinations. In this analysis tumour marker assay with CE or PI in one or more markers, unexplained by any concomitant transient or chronic benign pathology, that is for unknown reasons, was defined as a probably positive test. In both analyses tumour marker assay with normal or IEV was defined as a probably negative test. In the further analysis also CE or PI in one or more markers likely due to a clear concomitant benign pathology was defined as a probably negative test.

A probably positive test, according to whether it was or was not confirmed by monitoring to death or by the development of a definite clinical-imaging course after the 
initial result, was defined as true or false positive respectively. A probably negative test, according to whether it was or was not confirmed by monitoring by at least one year without any clinical-imaging sign of relapse, was defined as true or false negative.

Because one of 18 relapsed patients recurred twice during the follow-up (see the Result section), statistical analysis was performed considering 19 relapses.

\section{Results}

During the post-operative follow-up distant metastases occurred in $18(6.7 \%)$ of the 268 disease-free patients. Nine $(50 \%)$ of these 18 patients were postmenopausals. One of them recurred twice. In this patient multiple bone and liver metastases were found 9 months after she had been rendered disease-free with surgical removal of a single secondary liver nodule. Therefore, as total, there were 19 relapses. The organs initially involved in the relapse were: bone (8), viscera (8), soft tissue (1), bone and viscera (2). The number of the lesions was: $<3,>3<10$ and $>10$ in 8,6 and 5 relapses respectively.

Sensitivity of each tumour marker: CEA,TPA,CA15.3 and MCA ( $\geq 11$ or $\geq 15 \mathrm{U} / \mathrm{mL}$ cut-off value) (Table 1 )

MCA cut-off value $\geq 11 \mathrm{U} / \mathrm{mL}$. MCA, CEA, CA15.3 and TPA were the first finding in 2 to 13 relapses. In 6 relapses for MCA and in 1 for CEA the tumour marker increase was the only sign. In 7 relapses for MCA, in 1 for CEA and in all 5 and 6 relapses for TPA and CA15.3 respectively the tumour marker increase was concomitant with the increase of other markers and/or with clinical or instrumental findings. The mean lead time from the tumour marker increase to the appearance of clinical and/or instrumental signs of the relapse ranged from $2 \pm 2.8$ for CEA to $7.1 \pm 6.8$ months for MCA. BS alone or combined with tumour markers (one or more) was the first finding of relapse more frequently than LE or clinical symptoms (4 vs 1 and 2 relapses respectively). In these instances the mean lead time from the appearance of clinical and/or instrumental signs to tumour marker increase ranged from 0 for LE to $3 \pm 4.2$ months for clinical symptoms.

MCA cut-off value $\geq 15 \mathrm{U} / \mathrm{mL}$. MCA, CEA, TPA and CA15.3 were the first finding in 3 to 10 relapses. In 1 relapse for MCA and for CEA the tumour marker increase was the only sign. In 9 relapses for MCA, in 2 for CEA and in all 8 and 6 relapses for TPA and CA15.3 respectively the tumour marker increase was concomitant with the increase of other markers and/or with clinical or instrumental findings. The mean lead time from tumour marker increase to the appearance of clinical and/or instrumental signs of the relapse ranged from $2.9 \pm 4$ for TPA to $7.3 \pm$ 9.4 months for CEA. Again, BS alone or combined with tumour marker increase was the first finding of relapse more frequently than LE or clinical symptoms (4 vs 2 and 3 relapses respectively). In these instances the mean lead time from the appearance of clinical and/or instrumental signs to tumour marker increase ranged from $2 \pm 3.5$ for clinical symptoms to $5.5 \pm 7.8$ months for LE.

Table I: Sensitivity of MCA (* $\geq$ II or $* * \geq 15 \mathrm{U} / \mathrm{mL}$ cut-off values), CEA, TPA, CAI 5.3 in the early detection of 19 relapses during an intensive post-operative follow-up with tumour markers.

\begin{tabular}{|c|c|c|c|c|c|}
\hline \multirow{3}{*}{ Tumour marker type } & \multicolumn{5}{|c|}{ CE or PI of tumour markers as the initial pathological finding of relapse } \\
\hline & \multirow[t]{2}{*}{ aRelapses (total, $\mathrm{n}$ ) } & \multirow[t]{2}{*}{ Sensitivity (\%) } & \multicolumn{2}{|c|}{ Tumour marker increase } & \multirow[t]{2}{*}{ Lead time (months) $\mathrm{m}+\mathrm{sd}$ (range) } \\
\hline & & & type & $\mathrm{n}$ & \\
\hline *MCA & 13 & 68 & $\begin{array}{l}\text { CE } \\
\mathrm{PI}\end{array}$ & $\begin{array}{l}8 \\
5\end{array}$ & $\begin{array}{c}7.1 \pm 6.8 \\
(0-22)\end{array}$ \\
\hline CEA & 2 & 10 & $\begin{array}{l}\text { CE } \\
\mathrm{PI}\end{array}$ & $\begin{array}{l}0 \\
2\end{array}$ & $\begin{array}{c}2.0 \pm 2.8 \\
(0-4)\end{array}$ \\
\hline TPA & 5 & 26 & $\begin{array}{l}\text { CE } \\
\mathrm{PI}\end{array}$ & $\begin{array}{l}3 \\
2\end{array}$ & $\begin{array}{c}3.2 \pm 4.4 \\
(0-9)\end{array}$ \\
\hline CAI5.3 & 6 & 32 & $\begin{array}{l}\text { CE } \\
\mathrm{PI}\end{array}$ & $\begin{array}{l}5 \\
1\end{array}$ & $\begin{array}{c}4.7 \pm 5.8 \\
(0-14)\end{array}$ \\
\hline$* *$ MCA & 10 & 53 & $\begin{array}{l}\text { CE } \\
\mathrm{PI}\end{array}$ & $\begin{array}{l}7 \\
3\end{array}$ & $\begin{array}{c}6.5 \pm 6.8 \\
(0-18)\end{array}$ \\
\hline CEA & 3 & 16 & $\mathrm{PI}$ & 3 & $\begin{array}{c}7.3 \pm 9.4 \\
(0-18)\end{array}$ \\
\hline TPA & 8 & 42 & $\begin{array}{l}\text { CE } \\
\mathrm{PI}\end{array}$ & $\begin{array}{l}6 \\
2\end{array}$ & $\begin{array}{c}2.9 \pm 4.0 \\
(0-9)\end{array}$ \\
\hline CAI5.3 & 6 & 32 & $\begin{array}{l}\text { CE } \\
\mathrm{PI}\end{array}$ & $\begin{array}{l}5 \\
1\end{array}$ & $\begin{array}{c}4.7 \pm 5.8 \\
(0-14)\end{array}$ \\
\hline
\end{tabular}

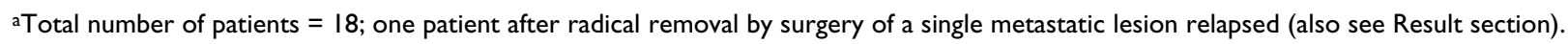

Therefore, percentages are calculated on 19 relapses; $\mathrm{CE}=$ constant elevation; $\mathrm{PI}=$ progressive increase. 
Sensitivity of different tumour marker associations: MCA ( $\geq 11$ or $\geq 15 \mathrm{U} / \mathrm{mL}$ cut-off values) - CA15.3 and CEATPA-CA15.3 panels (Table 2)

MCA cut-off value $\geq 11 \mathrm{U} / \mathrm{mL}$. MCA-CA15.3 and CEATPA-CA15.3 panels were the first finding of 13 and 10 relapses respectively. In 6 relapses for the former association and in 2 for the latter the tumour marker increase was the only sign while in the remaining it was the first finding of relapse concomitant with clinical and/or instrumental examinations. BS alone or combined with tumour marker increase was the first finding in 4 relapses, while it occurred in 1 relapse for LE and in 2 relapses for clinical symptoms. In these instances the mean lead time from the appearance of clinical and/or instrumental signs to tumour marker increase ranged from 0 for LE to $3 \pm 4.2$ months for clinical symptoms.

MCA cut-off value $\geq 15 \mathrm{U} / \mathrm{mL}$. CEA-TPA-CA15.3 panel and MCA-CA15.3 association were the first finding of 14 and 11 relapses respectively. In 2 relapses for the former association and in 1 relapse for the latter the tumour marker increase was the only sign while in the remaining it was the first finding of relapse concomitant with clinical and/or instrumental examinations. Eight of the 14 recurrences "early" detected using CEA-TPA-CA15.3 tumour marker panel presented as a single lesion (5) or confined to bony skeleton (3). Three of the 5 recurrences that presented as single lesions involved bony skeleton and the 2 remaining liver. Therefore, "early" detected recurrences that presented as a single lesion or limited to bony skeleton were $26 \%$ and $16 \%$ respectively of the 19 relapses. BS alone or combined with tumour marker increase was the first finding in 4 relapses, while it occurred in 2 relapses for LE and in 3 relapses for clinical symptoms. In these instances the mean lead time from the appearance of clin- ical and/or instrumental signs to tumour marker increase ranged from $1.5 \pm 3$ for BS to $5.5 \pm 7.8$ months for LE.

With both MCA cut-off values, no significant difference occurred between the lead time of MCA-CA15.3 and CEATPA-CA15.3 panels (p n.s., unpaired t test).

Specificity of each tumour marker: CEA, TPA, CA15.3, MCA

CE and/or PI occurred in 11 patients for CEA and in 8 patients for CA15.3. Diabetes and/or hepatic steatosis (5 patients), smoking (4 patients), miscellanea (1 patient) for CEA, chronic liver failure (2 patients), diabetes and/or hepatic steatosis (5 patients), hepatic cyst or angioma (1 patient) for CA15.3 were the concomitant conditions probably responsible for these two kinds of tumour marker increase. Significant increases for unknown reasons (false positives) occurred in no patient for CA15.3 and in 1 patient $(0.4 \%)$ for CEA. CE and/or PI occurred in 69 patients for TPA and in 144 patients or in 68 patients for MCA with $\geq 11 \mathrm{U} / \mathrm{mL}$ or $\geq 15 \mathrm{U} / \mathrm{mL}$ cut-off value respectively. Chronic liver failure ( 7 patients), diabetes and/or hepatic steatosis (36 patients), acute inflammation of upper airways ( 6 patients) for TPA, chronic liver failure (6 patients), diabetes and/or hepatic steatosis (54 patients), acute joint inflammation (9 patients), acute inflammation of upper airways (9 patients), hepatic cyst and/or angioma (9 patients), miscellanea (7 patients) for MCA with $\geq 11 \mathrm{U} / \mathrm{mL}$ cut-off value, chronic liver failure (5 patients),diabetes and/or hepatic steatosis (23 patients), acute joint inflammation (5 patients), hepatic cyst and/or angioma ( 6 patients) for MCA with $\geq 15 \mathrm{U} / \mathrm{mL}$ cut-off value were the concomitant conditions more often probably responsible for these two different kinds of tumour marker increase. Significant increases for unknown rea-

Table 2: Sensitivity of MCA (* $\geq 11$ or $* * \geq 15 \mathrm{U} / \mathrm{mL}$ cut-off values)-CAI5.3 and CEA-TPA-CAI5.3 panels in the early detection of 19 relapses during an intensive post-operative follow-up with tumour markers.

\begin{tabular}{|c|c|c|c|c|c|}
\hline \multirow{3}{*}{ Tumour marker association } & \multicolumn{5}{|c|}{$\mathrm{CE}$ or $\mathrm{PI}$ of tumour markers as the initial pathological finding of relapse } \\
\hline & \multirow{2}{*}{$\begin{array}{l}\text { aRelapses } \\
\text { (total, n) }\end{array}$} & \multirow{2}{*}{$\begin{array}{l}\text { Sensitivity } \\
(\%)\end{array}$} & \multicolumn{2}{|c|}{ Tumour marker increase } & \multirow{2}{*}{$\begin{array}{l}\text { Lead time (months) } \\
\qquad \mathrm{m}+\mathrm{sd} \text { (range) }\end{array}$} \\
\hline & & & type & $\mathrm{n}$ & \\
\hline *MCA-CAI 5.3 & 13 & 68 & $\begin{array}{l}\text { CE } \\
\mathrm{PI}\end{array}$ & 85 & $\begin{array}{c}7.1 \pm 6.8 \\
(0-22)\end{array}$ \\
\hline CEA-TPA-CAI5.3 & 10 & 53 & $\begin{array}{l}\text { CE } \\
\mathrm{PI}\end{array}$ & 55 & $\begin{array}{c}3.9 \pm 4.9 \\
(0-14)\end{array}$ \\
\hline **MCA-CAI 5.3 & 11 & 58 & $\begin{array}{l}\mathrm{CE} \\
\mathrm{PI}\end{array}$ & 83 & $\begin{array}{c}5.9 \pm 6.7 \\
(0-18)\end{array}$ \\
\hline CEA-TPA-CAI5.3 & 14 & 74 & $\begin{array}{l}\mathrm{CE} \\
\mathrm{PI}\end{array}$ & 86 & $\begin{array}{c}4.6 \pm 5.9 \\
(0-18)\end{array}$ \\
\hline
\end{tabular}

aTotal number of patients = 18; one patient after radical removal by surgery of a single metastatic lesion relapsed (also see Result section). Therefore, percentages are calculated on 19 relapses; $\mathrm{CE}=$ constant elevation; $\mathrm{PI}=$ progressive increase. 
sons (falsely positives) were found in 10 (4\%), 43 $(17.2 \%)$ and $20(8 \%)$ patients for TPA, MCA with $\geq 11 \mathrm{U} /$ $\mathrm{mL}$ and MCA with $\geq 15 \mathrm{U} / \mathrm{mL}$ cut-off value respectively. Therefore, specificity was 100\%, 100\%, 96\%, 83\% and $92 \%$ for CEA, CA15.3, TPA and MCA $(\geq 11 \mathrm{U} / \mathrm{mL}$ or $\geq 15$ $\mathrm{U} / \mathrm{mL}$ cut-off value) respectively, when an accurate history was taken into account. Without an accurate history, specificity was $96 \%, 97 \%, 72 \%, 42 \%$ and $73 \%$ for CEA, CA15.3, TPA and MCA $(\geq 11 \mathrm{U} / \mathrm{mL}$ or $\geq 15 \mathrm{U} / \mathrm{mL}$ cut-off value) respectively.

Specificity of different tumour marker associations: MCA ( $\geq 11 \mathrm{U} / \mathrm{mL}$ or $\geq 15 \mathrm{U} / \mathrm{mL}$ cut-off value) - CA15.3 and CEA-TPA-CA15.3

CE and/or PI of CEA-TPA-CA15.3 panel occurred in 77 patients. Diabetes and/or hepatic steatosis (40 patients), chronic liver failure ( 7 patients), acute inflammation of upper airways (5 patients) were the concomitant conditions probably responsible for the tumour markers' increase.

When MCA cut-off value was $\geq 11 \mathrm{U} / \mathrm{mL}, \mathrm{CE}$ and/or PI of MCA-CA15.3 association occurred in 151 patients. Diabetes and/or hepatic steatosis (58 patients), hepatic cyst and/or angioma (12 patients), acute joint inflammation (10 patients), acute inflammation of upper airways ( 9 patients) were the concomitant conditions more often probably responsible for these two different kinds of tumour marker increase.

When MCA cut-off value was $\geq 15 \mathrm{U} / \mathrm{mL}, \mathrm{CE}$ and/or PI of MCA-CA15.3 association occurred in 72 patients. Diabetes and/or hepatic steatosis (24 patients), hepatic cyst and/or angioma ( 7 patients), chronic liver failure ( 6 patients) were the concomitant conditions more often probably responsible for these two different kinds of tumour marker increase. Significant increases for unknown reasons (falsely positives) occurred in 11 (4.4\%), 41 (16.4\%) and 19 (7.6\%) patients for CEA-TPACA15.3 panel, MCA-CA15.3 association with cut-off value $\geq 11 \mathrm{U} / \mathrm{mL}$ and MCA-CA15.3 association with cut-off value $\geq 15 \mathrm{U} / \mathrm{mL}$ respectively.

Therefore, specificity was $96 \%, 84 \%$ and $92 \%$ for CEATPA-CA15.3 and MCA ( $\geq 11 \mathrm{U} / \mathrm{mL}$ or $\geq 15 \mathrm{U} / \mathrm{mL}$ cut-off value) - CA15.3 associations respectively, when an accurate history was taken into account. Without an accurate history, specificity was $69 \%, 40 \%$ and $71 \%$ for CEA-TPACA15.3 and MCA ( $\geq 11 \mathrm{U} / \mathrm{mL}$ or $\geq 15 \mathrm{U} / \mathrm{mL}$ cut-off value) - CA15.3 associations respectively.

Reliability of MCA ( $\geq 11 \mathrm{U} / \mathrm{mL}$ or $\geq 15 \mathrm{U} / \mathrm{mL}$ cut-off values) - CA15.3 and CEA-TPA-CA15.3 tumour marker panels with and without an accurate history (Table 3)
MCA cut-off value $\geq 11 \mathrm{U} / \mathrm{mL}$. When dynamic evaluation of tumour marker associations was considered with an accurate history and laboratory examinations, specificity, accuracy and PPV of CEA-TPA-CA15.3 tumour marker panel were higher than the corresponding of MCACA15.3 association. The opposite occurred for sensitivity and NPV. When dynamic evaluation of tumour markers was considered alone, specificity, accuracy, NPV and PPV of CEA-TPA-CA15.3 tumour marker panel were higher than the corresponding of MCA-CA15.3 association. The opposite occurred for sensitivity.

MCA cut-off value $\geq 15 \mathrm{U} / \mathrm{mL}$. When dynamic evaluation of tumour marker associations was considered with an accurate history and laboratory examinations, sensitivity, specificity, accuracy, NPV and PPV of CEA-TPA-CA15.3 tumour marker panel were higher than the corresponding of MCA-CA15.3 association. When dynamic evaluation of tumour markers was considered alone, sensitivity, accuracy, NPV and PPV of CEA-TPA-CA15.3 tumour marker panel were higher than the corresponding of MCACA15.3 association. The opposite occurred for specificity (Table 3).

\section{Discussion}

MCA sensitivity for "early" detection of relapses, either with $\geq 11$ or $\geq 15 \mathrm{UI} / \mathrm{mL}$ cut-off value, was higher than that of CEA or CA15.3 or TPA. With regard to CEA, TPA and CA15.3, CA15.3 (with MCA cut-off value $\geq 11 \mathrm{UI} /$ $\mathrm{mL}$ ) and TPA (with MCA cut-off value $\geq 15 \mathrm{UI} / \mathrm{mL}$ ) were more sensitive than both remaining indicators. However, only with $\geq 11 \mathrm{U} / \mathrm{mL}$ MCA cut-off value, sensitivity increased slightly (from 53\% to 58\%) after the association of MCA with CA15.3, while as we [4,7,9-11] and others $[22,26,33]$ previously reported, it occurred significantly when TPA was associated with CA15.3 and CEA (Tables 1, 2 ). In other studies a range of MCA sensitivity similar to CA15.3 and no significant increase in sensitivity when MCA was combined with CA15.3 were found $[14,27,34,35]$. These findings and our results suggest that, although MCA and CA15.3 recognise distinct epitopes on the same molecule [36], in metastatic breast cancer cells MCA expression almost completely overlaps that of CA15.3, while it partially occurs among CEA, TPA and CA15.3.

Total rate of significant increases of MCA was similar to or higher ( $\geq 11$ or $\geq 15 \mathrm{UI} / \mathrm{mL}$ cut-off value respectively) than that of TPA and it was higher than that of CEA and CA15.3. Consequently, significant increases of MCACA15.3 association occurred more frequently (60\% vs $31 \%$ ) or similarly (29\% vs $31 \%$ ) to those of CEA-TPACA15.3 tumour marker panel. This finding does not confirm that MCA specificity is similar to or higher than that of CEA and CA15.3 [17,20-23]. Moreover, our results 
Table 3: Comparison between reliability of MCA $\left(* \geq 1 / \mathrm{U} / \mathrm{mL}\right.$ or ${ }^{* *} \geq 15 \mathrm{U} / \mathrm{mL}$ cut-off values) - CA I5.3 and CEA-TPA-CAI5.3 associations with or without an accurate history during intensive post-operative follow-up with tumour markers of 289 breast cancer patients.

\begin{tabular}{|c|c|c|c|c|}
\hline \multirow[b]{2}{*}{ Reliability } & \multicolumn{4}{|c|}{ Tumour marker panel } \\
\hline & CEA-TPA-CAI5.3 & *MCA-CAI5.3 & CEA-TPA-CAI 5.3 & **MCA-CAI5.3 \\
\hline Sensitivity \% & $53(53)$ & $68(68)$ & $74(74)$ & $58(58)$ \\
\hline Specificity \% & $96(69)$ & $84(40)$ & $96(69)$ & $92(7 I)$ \\
\hline Accuracy \% & $92.5(68)$ & $82.5(42)$ & $94(69.5)$ & $90(4 I)$ \\
\hline NPV \% & $96(95)$ & $97(94)$ & $98(97)$ & $97(92)$ \\
\hline PPV\% & $48(I I)$ & $24(8)$ & $56(15)$ & $37(7)$ \\
\hline
\end{tabular}

Numbers into brackets refer to dynamic evaluation of tumour markers without an accurate history and laboratory examinations.

show that in non relapsed patients the aspecific reasons probably responsible for MCA increase are the same as for TPA. The addition, at each control visit, of an accurate history and laboratory examinations to the dynamic evaluation of tumour markers increased their specificity while sensitivity remained unchanged. In fact, in $8(42 \%)$ of the 19 relapses, a concomitant benign pathology occurred. Nevertheless, in all of them CE and/or PI in one or more markers could be referred to a pending relapse rather than to the concomitant benign pathology. Conversely, among the non relapsed patients those falsely suspected with all evaluated tumour markers particularly MCA, TPA and their combinations strongly decreased. In fact, CE and/or PI, unexplained by a clear concomitant benign pathology, ranged from $0 \%$ for CA15.3 to $17 \%$ for MCA with $\geq 11$ cut-off value. When MCA cut-off value was $\geq 11 \mathrm{UI} / \mathrm{mL}$, MCA-CA15.3 association showed higher sensitivity and NPV but lower specificity, accuracy and PPV than CEATPA-CA15.3 panel. When MCA cut-off value was $\geq 15 \mathrm{UI} /$ $\mathrm{mL}$, sensitivity, specificity, accuracy, NPV and PPV of CEATPA-CA15.3 panel were higher than those of MCACA15.3 association (Table 3 ).

Being confined to bony skeleton is considered a favourable prognostic factor for metastatic disease $[37,38]$. Also it has been shown that a single lesion, i.e. minimal metastatic disease called "stage IV oligometastatic disease", amenable to local therapy (surgery and/or radiation) followed by high dose chemotherapy is considered another different favourable condition $[39,40]$. In these metastatic patients with oligometastatic disease or disease limited to bony skeleton, median overall survival 2-3 times longer than in general metastatic population is expected. In a general metastatic population at the presentation bony skeleton as dominant site and oligometastatic disease have been reported to involve about 15\% [41-43] and 5$10 \%[38,44,45]$ of patients respectively. Therefore, in this study a post-operative follow-up with an appropriate use of CEA-TPA-CA15.3 tumour marker panel also "early" detected a relatively high percentage of relapsed patients with limited metastatic disease and more favourable prognosis.

\section{Conclusion}

In conclusion, data from this study point out that at both the evaluated cut-off values serum MCA sensitivity is higher than that of CEA, TPA and CA15.3. However, MCA specificity is the lowest or similar to that of TPA and they are both much lower than those of CEA and CA15.3. Despite a higher sensitivity, this low specificity represents an important limitation for a meaningful clinical applicability of MCA and TPA as single marker. Furthermore they suggest that overall CEA-TPA-CA15.3 panel is more suitable than MCA-CA15.3 association for an intensive postoperative follow-up of breast cancer patients with tumour markers and that an intensive post-operative follow-up with CEA-TPA-CA15.3 panel can early detect relapsing patients with more favourable prognosis. These findings further urge the need for randomised clinical trial to assess whether an "early signalling" and treatment of distant metastases with an appropriate use of serum tumour markers also can significantly improve OS. Moreover, a detailed cost-efficacy ratio analysis from these trials will permit to draw definite conclusions about the usefulness of serum tumour markers for a routine use.

\section{Abbreviations}

CEA: Carcinoembryonic antigen

TPA: tissue polypeptide antigen

CA15.3: breast cancer associated 115D8/DF3 antigen

MCA: mucin-like carcinoma associated antigen

DFS: disease-free survival

OS: overall survival

$\mathrm{N}$ : axillary lymph-node involvement 
PgR: progesterone receptor

Sx-ray: skeletal x-ray

BS: Bone scintigraphy BS

LE: liver echography

CXR: chest x-ray

IEV: isolated elevated value

CE: constant elevation

PI: progressive increase

FP: false positive

FN: false negative

TP: true positive

TN: true negative.

NPV: negative predictive value

PPV: positive predictive value

\section{Competing interests}

The author(s) declare that they have no competing interests.

\section{Authors' contributions}

AN was responsible for study design, data analysis and preparation of manuscript; GT, AC, PF, LA, MC, PM for data analysis and preparation of manuscript; MRM for laboratory data and data analysis.

\section{References}

I. Smith TJ, Davidson NE, Schapira DV, Grunfeld E, Muss HB, Vogel VG 3rd, Somerfield MR: American Society of Clinical Oncology 1998 update of recommended breast cancer surveillance guidelines. J Clin Oncol 1999, I 7:1080-1082.

2. The GIVIO Investigators: Impact of follow-up testing on survival and health related quality of life in breast cancer patients. JAMA 1994, 27 I: I587-1592.

3. Rosselli Del Turco M, Palli D, Cariddi A, Ciatto S, Pacini P, Distante $\mathrm{V}$ : Intensive diagnostic follow-up after treatment of primary breast cancer. A randomized trial. JAMA 1994, 27 I:1593-1597.

4. Nicolini A, Carpi A: Postoperative follow-up of breast cancer patients: overview and progress in the use of tumor markers. Tumor Biol 2000, 2 1:235-248.

5. Jager W: The early detection of disseminated (metastasized) breast cancer by serial tumour marker measurements. Eur J Cancer Prev : 133-9. 1993 Nov;2

6. Kovner F, Merimsky O, Hareuveni M, Wigler N, Chaitchik S: Treatment of disease-negative but mucin-like carcinoma-associated antigen-positive breast cancer patients with tamoxifen: preliminary results of a prospective controlled randomized trial. Cancer Chemother Pharmacol 1994, 35(I):80-3.
7. Nicolini A, Anselmi L, Michelassi C, Carpi A: Prolonged survival by "early" salvage treatment of breast cancer patients: a retrospective 6-year study. Br J Cancer 1997, 76: I I06-I I II.

8. Seregni E, Coli A, Mazzucca N: Italian Group RIA-IRMA Test, Italian Association of Nuclear Medicine: Circulating tumour markers in breast cancer. Eur J Nucl Med Mol Imaging 2004, 3 I (I):SI 5-22.

9. Nicolini A, Ferrari P, Sagripanti A, Carpi A: The role of tumour markers in predicting skeletal metastases in breast cancer patients with equivocal bone scintigraphy. Br J Cancer 1999, 79: |443- 447.

10. Nicolini A, Carpi A, Ferrari P, Anselmi L, Spinelli C, Conte M, Miccoli $P$ : The role of tumour markers in improving the accuracy of conventional chest x-ray and liver echography in the postoperative detection of thoracic and liver metastases from breast cancer. BrJ Cancer 2000, 83: |4I2-14I7.

II. Nicolini A, Carpi A, Ferrari P, Pieri L: Utility of a serum tumour marker panel in the post-operative follow-up of breast cancer patients with equivocal conventional radiological examinations. Tumor Biol 2003, 24:275-280.

12. Eskelinen M, Hippelainen M, Salmela E, Paajanen H, Alhava E, Syrjanen $\mathrm{K}$ : A decision support system for predicting a recurrence of breast cancer; a prospective study of serum tumour markers TAG I 2, CA I 5-3 and MCA. Anticancer Res 1992, I 2: |439-| 442.

13. Nicolini A, Ferdeghini M, Colombini C, Carpi A: Evaluation of serum CA549, CA M26 and CA M29 levels in the post-operative follow-up of breast cancer patients. J Nucl Med Allied Sci 1990, 34:309-313.

14. De Wit R, Hoek FJ, Bakker PJ, Veenhof $\mathrm{CH}$ : A comparison of CA549 with CA I5-3 and MCA in patients with metastatic breast cancer. Ann Oncol 1992, 3:3|4-315.

15. Kopczynsky Z, Thielemann A: The value of tissue polypeptide specfic antigen TPS determination in serum of women with breast cancer comparison to mucin-like associated antigen MCA and CA I5-3 antigen. Eur J Gynaecol Oncol 1998, I 9:503-507.

16. Nicolini A, Carpi A, Di Marco G, Giuliani L, Giordani R, Palla S: A rational postoperative follow-up with carcinoembryonic antigen, tissue polypeptide antigen, and urinary hydroxyproline in breast cancer patients. Cancer 1989, 63:2037-2046.

17. Cooper EH, Forbes MA, Hancock AK, Price JJ, Parker D: An evaluation of mucin-like carcinoma associated antigen (MCA) in breast cancer. Br J Cancer 1989, 59:797-800.

18. Nicolini A, Colombini C, Luciani L, Carpi A: Evaluation of serum CA 15-3 determination with CEA and TPA in the post-operative follow-up of breast cancer patients. $\mathrm{Br} J$ Cancer I99I, 64:154-158.

19. Gion M, Daidone MG: Circulating biomarkers from tumour bulk to tumour machinery: promises and pitfalls. Eur J Cancer 2004, 40:2613-2622.

20. Merimsky O, Inbar M, Hareuveni M, Witenberg B, Wolman $Y$, Chaitchik S: Serial serum MCA measurements in the followup of breast cancer patients. Eur J Cancer I99 I, 27: |440- |444.

21. Ammon A, Eiffert H, Alhusen R, Weber M, Rumelin B, Groh E, Bartsch H, Marschner N, Nagel GA, Krieger G: Mucin-like carcinomaassociated antigen: sensitivity and specificity in metastatic breast cancer. Onkologie 1990, I3:210-214.

22. Pectasides D, Pavlidis N, Gogou L, Antoniou F, Nicolaides C, Tsikalakis D: Clinical value of CA I5-3, mucin-like carcinoma-associated antigen, tumor polypeptide antigen, and carcinoembryonic antigen in monitoring early breast cancer patients. Am J Clin Oncol 1996, 19:459-464.

23. Miserez AR, Gunes I, Muller-Brand J, Walther E, Fridrich R, Macke H: Clinical value of a mucin-like carcinoma-associated antigen in monitoring breast cancer patients in comparison with CA 15-3. Eur J Cancer I991, 27:|26-I3I.

24. Jezersek B, Cervek J, Rudolf Z, Novakovic S: Clinical evaluation of potential usefulness of CEA, CA 15-3, and MCA in follow-up of breast cancer patients. Cancer Lett 1996, 20: I 37-I44.

25. Dixon AR, Price MR, Hand CW, Sibley PE, Selby C, Blamey RW: Epithelial mucin core antigen (EMCA) in assessing therapeutic response in advanced breast cancer-a comparison with CA 1 5.3. Br J Cancer 1993, 68:947-949.

26. Gion M, Mione R, Gatti C, Dittadi R, Leon A, Nascimben O, Pizzorno $B$, Bruscagnin $G$ : Is tissue polypeptide antigen still a useful 
tumor marker in breast carcinoma? Comparison with CAI5.3 and MCA. Tumori 1990, 76:360-364.

27. Martoni A, Zamagni C, Bellanova B, Zanichelli L, Vecchi F, Cacciari N, Strocchi E, Pannuti F: CEA, MCA, CA I5.3 and CA 549 and their combinations in expressing and monitoring metastatic breast cancer: a prospective comparative study. Eur J Cancer 1995, 31:1615-1621.

28. Goldhirsch A, Glick JH, Gelber RD, Coates AS, Thurlimann B, Senn $\mathrm{HJ}$ : Panel members. Meeting highlights: international expert consensus on the primary therapy of early breast cancer 2005. Ann Oncol 2005, 16(10):1569-83.

29. Clark GM, Wenger CR, Beardslee S, Owens MA, Pounds G, Oldake $T$, Vendely P, Pandian MR, Harrington D, McGuire WL: How to integrate steroid hormone receptor, flow cytometric, and other prognostic information in regard to primary breast cancer. Cancer 7 I (6 Suppl):2157-62. 1993 Mar 15

30. Ruder AM, Lubin F, Wax Y, Geier A, Alfundary E, Chetrit A: Estrogen and progesterone receptors in breast cancer patients. Epidemiologic characteristics and survival differences. Cancer 64(I): 196-202. I 989 Jul I

31. Ciatto S, Pacini P, Andreoli C, Cecchini S, lossa A, Grazzini G, Buranelli $F$, Campa T, Costa A, Magni A: Chest X-ray survey in the follow-up of breast cancer patients. $\mathrm{Br} J$ Cancer 1989, 60:102-103.

32. Kokko R, Hakama M, Holli K: Role of chest X-ray in diagnosis of the first breast cancer relapse: a randomized trial. Breast $\mathrm{Can}$ cer Res Treat 2003, 81:33-39.

33. Barak M, Steiner M, Finkel B, Abrahamson J, Antal S, Gruener N: CA15.3, TPA and MCA as markers for breast cancer. Eur J Cancer 1990, 26:577-580

34. Garcia MB, Blankenstein MA, van der Wall E, Nortier JW, Schornage $\mathrm{JH}$, Thijssen JH: Comparison of breast cancer mucin (BCM) and CA 15-3 in human breast cancer. Breast Cancer Res Treat 1990, 17:69-76.

35. Silver HK, Archibald BL, Ragaz J, Coldman AJ: Relative operating characteristic analysis and group modeling for tumor mark ers: comparison of CA 15.3, carcinoembryonic antigen, and mucin-like carcinoma-associated antigen in breast carcinoma. Cancer Res 1991, 5 I: 1904-1909.

36. Daly L, Ferguson J, Cram GP Jr, Hars V, George SL, McCarty KS Jr, Bast RC Jr: Comparison of a novel assay for breast cancer mucin to CAI5-3 and carcinoembryonic antigen. J Clin Oncol 1992, 10:1057-1065.

37. Sherry MM, Greco FA, Johnson DH, Hainsworth JD: Metastatic breast cancer confined to the skeletal system. An indolent disease. Am J Med 1986, 8I:38I-386.

38. Koizumi M, Yoshimoto M, Kasumi F, Ogata E: Comparison between solitary and multiple skeletal metastatic lesions of breast cancer patients. Ann Oncol 2003, 14:1234-1240.

39. Hortobagyi GN: Can we cure limited metastatic breast cancer? J Clin Oncol 2002, 20:620-623.

40. Nieto Y, Nawaz S, Jones RB, Shpall EJ, Cagnoni PJ, McSweeney PA Baron A, Razook C, Matthes S, Bearman SI: Prognostic model for relapse after high-dose chemotherapy with autologous stem-cell transplantation for stage IV oligometastatic breast cancer. I Clin Oncol 2002, 20:707-718.

4I. Vici P, Colucci G, Gebbia V, Amodio A, Giotta F, Belli F, Conti F, Gebbia N, Pezzella G, Valerio MR, Brandi M, Pisconti S, Durini E, Giannarelli D, Lopez M: First-line treatment with epirubicin and vinorelbine in metastatic breast cancer. J Clin Oncol 20(II):2689-94. 2002 Jun I

42. Falkson G, Gelman RS, Pandya KJ, Osborne CK, Tormey D, Cummings FJ, Sledge GW, Abeloff MD: Eastern Cooperative Oncology Group randomized trials of observation versus maintenance therapy for patients with metastatic breast cancer in complete remission following induction treatment. J Clin Oncol 1998, 16(5):1669-76.

43. Fleming GF, Kugler JW, Hoffman PC, Ansari R, Bitran JD, Klepsch A, Malone D, Fasanmade AA, Ratain MJ, Vokes EE: Phase II trial of paclitaxel and topotecan with granulocyte colony-stimulating factor support in stage IV breast cancer. J Clin Oncol 1998, I 6(6):2032-7.

44. Singletary SE, Walsh G, Vauthey JN, Curley S, Sawaya R, Weber KL Meric $F$, Hortobagyi $G N$ : A role for curative surgery in the treatment of selected patients with metastatic breast cancer. Oncologist 2003, 8(3):24I-5I.
45. Rivera E, Holmes FA, Buzdar AU, Asmar L, Kau SW, Fraschini G, Walters R, Theriault RL, Hortobagyi GN: Fluorouracil, doxorubicin, and cyclophosphamide followed by tamoxifen as adjuvant treatment for patients with stage IV breast cancer with no evidence of disease. Breast J 2002, 8(I):2-9.

\section{Pre-publication history}

The pre-publication history for this paper can be accessed here:

http://www.biomedcentral.com/1471-2407/6/269/pre pub
Publish with Bio Med Central and every scientist can read your work free of charge

"BioMed Central will be the most significant development for disseminating the results of biomedical research in our lifetime. "

Sir Paul Nurse, Cancer Research UK

Your research papers will be:

- available free of charge to the entire biomedical community

- peer reviewed and published immediately upon acceptance

- cited in PubMed and archived on PubMed Central

- yours - you keep the copyright 